\title{
Tspan9 inhibits the proliferation, migration and invasion of human gastric cancer SGC7901 cells via the ERK1/2 pathway
}

\author{
PAI-YUN LI* , JING LV*, WEI-WEI QI, SHU-FEN ZHAO, LI-BIN SUN, \\ NING LIU, JIE SHENG and WEN-SHENG QIU
}

Department of Oncology, Affiliated Hospital of Qingdao University, Qingdao, Shandong 266003, P.R. China

Received January 1, 2016; Accepted February 17, 2016

DOI: $10.3892 / o r .2016 .4805$

\begin{abstract}
Tetraspanins are a heterogeneous group of 4-transmembrane proteins that recruit other cell surface receptors and signaling proteins into tetraspanin-enriched microdomains (TEMs). TEMs of various types are involved in the regulation of cell growth, migration and invasion of several tumor cell types, both as suppressors or promotors. Tetraspanin 9 (Tspan9, NET-5, PP1057), a member of the transmembrane 4 superfamily (TM4SF) of tetraspanins, reportedly regulates platelet function in concert with other platelet tetraspanins and their associated proteins. Our previous study demonstrated that Tspan9 is also expressed in gastric cancer (GC), but the role of Tspan9 in GC has not been well characterized. In this study, we investigated the influence of Tspan 9 on proliferation, migration and invasion of human gastric cancer SGC7901 cells using CCK-8 assay, cell cycle analysis, wound-healing assay and Transwell assay. Western blot analysis and ELISA assay were also performed to identify the potential mechanisms involved. The proliferation, migration and invasion of human gastric cancer SGC7901 cells were significantly inhibited by overexpression of Tspan9. In addition, Tspan9 downregulated the phosphorylation of extracellular signal-regulated kinases 1 and $2($ ERK1/2) and the secretion levels of proteins related to tumor metastasis, such as matrix metalloproteinase-9 (MMP-9) and urokinase plasminogen activator (uPA). Our study indicated that Tspan9 inhibited SGC7901 cell proliferation, migration and invasion through the ERK1/2 pathway.
\end{abstract}

\section{Introduction}

Gastric cancer (GC) is the second leading cause of cancerrelated death and the fourth most common malignant tumor

Correspondence to: Dr Wen-Sheng Qiu, Department of Oncology, Affiliated Hospital of Qingdao University, 16 Jiangsu Road, Qingdao, Shandong 266003, P.R. China

E-mail:wsqiuqd@163.com

*Contributed equally

Key words: gastric cancer, Tspan9, proliferation, migration, invasion, ERK1/2 pathway in the world (1). There are approximately 876,000 new cases diagnosed ( $8.7 \%$ of the total cancer cases), and 647,000 people die from the disease (10.4\% of cancer deaths) every year (2). However, the mechanisms underlying the development of GC remain largely unclear, and the genetic and molecular alterations in this malignant disease are not fully understood. Thus, good understanding of the molecular mechanisms underlying GC development and progression is urgently needed.

Tetraspanins are a large family of proteins (33 in mammals). These proteins have two small and large extracellular loops, four putative hydrophobic membrane-spanning domains and short amino- and carboxy-terminal cytoplasmic domains. The hypervariable region in the large extracellular domain of each tetraspanin can distinguish it from other members in this family (3). Extracellular domains allow tetraspanins to associate with a wide range of proteins by homo/heterotypic interactions (4). Tetraspanins form complexes termed tetraspanin-enriched membrane microdomains (TEMs) by interacting with other tetraspanins and with a variety of transmembrane and cytosolic proteins that are required for their function $(5,6)$. A multitude of biological processes are associated with tetraspanins, such as fertilization, parasite and viral infection, synaptic contacts at neuromuscular junctions, platelet aggregation, maintenance of skin integrity, immune response induction, metastasis suppression and tumor progression (7-9). Furthermore, changing the composition and localization of TEMs in response to external or internal stimuli reveals that TEMs are dynamic and flexible structures (10).

Tspan9, as a member of the tetraspanins, is a component of TEMs that include the collagen receptor GPVI (glycoprotein VI) and integrin $\alpha 6 \beta 1$, which suggest a role for Tspan9 in regulating platelet function in concert with other platelet tetraspanins and their associated proteins (11). However, the role of Tspan9 in GC cannot be predicted as tetraspanins own different abilities to promote or suppress carcinogenesis and cancer metastasis. For example, tetraspanins CD82 and CD9 mostly suppress tumor progression as they suppress motility and promote adherence to the surrounding matrix. Their expression is often reduced in late-stage human tumors. In contrast, tetraspanin CD151 and tetraspanin 8 are overexpressed in several tumors and appear to support tumor progression. CD151 regulates post-adhesion events, that is, cell spreading, migration and invasion including subsequent intravasation and formation of metastasis. Tetraspanin 8 
regulates cell motility and survival and is involved in the promotion of angiogenesis $(9,12,13)$.

Matrix metalloproteinases (MMPs) are highly expressed in almost all human cancers and so is urokinase plasminogen activator (uPA) $(14,15)$. The matrix metalloproteinase-9 (MMP-9) and UPA are responsible for the degradation of extracellular matrix components and play important roles in the process of cancer invasion and metastasis (16-18). In addition, many tetraspanins directly or indirectly regulate the expression and activity of MMP-9 or UPA. Thus, their regulation of MMP-9 or UPA indicates an anticancer therapeutic strategy $(9,19)$.

In the present study, we established a stable cell line with overexpressed Tspan9 to investigate the influence of Tspan9 on GC in vitro, looking at the functional effects and the possible involvement of Tspan9 in GC proliferation, migration and invasion by regulating the expression of MMP-9 and uPA through the ERK1/2 pathway.

\section{Materials and methods}

Cell culture and construction of the stable cell line overexpressing Tspan9. Human GC SGC7901 cells were obtained from the Central Laboratory of the Affiliated Hospital of Qingdao University and were cultured in Roswell Park Memorial Institute-1640 medium (RPMI-1640; Hyclone, Logan, UT, USA) supplemented with $10 \%$ fetal bovine serum (FBS; Gibco, Grand Island, NY, USA) at $37^{\circ} \mathrm{C}$ under $95 \%$ humidity and $5 \% \mathrm{CO}_{2}$ content. Cells were harvested in the logarithmic growth phase for use in the experiments described below. The day before transfection, the SGC7901 cells were seeded into 6-well plates to ensure $60 \%$ confluency at the time of transfection. Then the cells were transfected with lentivirus particles (Genechem Co., Ltd., Shanghai, China) coding for Tspan9 or the negative control (NC), respectively, at a multiplicity of infection of 10. Six hours later, the medium was replaced, and cells were cultured for an additional $24 \mathrm{~h}$ and processed for further experiments. Transduction efficiency, as determined by electron microscopy (GFP, x200 magnification) ranged between 80 and $90 \%$. The clones transfected with LV-Tspan9 were defined as the Tspan9 group, cells transfected with the negative control vectors were considered as the NC group, and the blank group included cells without transfection. Experiments were conducted with cell passages 3-20.

RNA extraction and real-time quantitative reverse transcription-PCR ( $q R T-P C R)$. Primer sequences (Invitrogen, Carlsbad, CA, USA) for human Tspan9 were forward, 5'-GAACGGGCT GCTATGAAAAGG-3' and reverse, 5'-CGTACTTCTTACCA GTCCGGT-3'. Total RNA was extracted from the cultured cells using mirVana ${ }^{\mathrm{TM}}$ PARIS $^{\mathrm{TM}}$ kit (Ambion, Carlsbad, CA, USA). The quality and quantity of the RNA were assessed with a spectrophotometer (NanoDrop Technologies, Wilmington, DE, USA) at 260 and $280 \mathrm{~nm}$ (A260/280). Then the RNA samples were reverse-transcribed with Transcriptor First Strand cDNA Synthesis kit (Roche, Mannheim, Germany). PCR conditions were as follows: $10 \mathrm{~min}$ at $95^{\circ} \mathrm{C}$; 45 cycles of $10 \mathrm{sec}$ at $95^{\circ} \mathrm{C}, 20 \mathrm{sec}$ at $60^{\circ} \mathrm{C}$ and $10 \mathrm{sec}$ at $72^{\circ} \mathrm{C}$. According to the manufacturer's protocol, each $20-\mu 1$ reaction system included $5 \mu \mathrm{l}$ of the cDNA reverse-transcribed as described above, $10 \mu \mathrm{l}$ of $2 \mathrm{X}$ FastStart Essential DNA Green Master (Roche), $2 \mu \mathrm{l}$ of 10X forward and reverse primers and $3 \mu \mathrm{l}$ of PCR grade water. Real-time PCR was performed on LightCycler ${ }^{\circledR} 96$ instrument (Roche), as fluorescence was detected at the end of each cycle. The relative amount of target genes was carried out using the $2^{-\Delta \Delta C t}$ method while GAPDH was used as internal control.

Western blot analysis. Cells were lysed on ice in RIPA buffer containing PMSF (Solarbio, Beijing, China) for $30 \mathrm{~min}$ vortexing every $10 \mathrm{~min}$, followed by centrifugation at $14,000 \mathrm{x} \mathrm{g}$ for $5 \mathrm{~min}$ at $4^{\circ} \mathrm{C}$ to remove cell debris. Then the supernatant was collected for determination of total protein concentration using the BCA protein assay kit (CWBIO, Beijing, China). Approximately 25-30 $\mu \mathrm{g}$ of protein was resolved by $12 \%$ sodium dodecyl sulfate-polyacrylamide gel electrophoresis and was transferred onto polyvinylidene difluoride membranes (Millipore, USA). The membranes were blocked in $5 \%$ bovine serum albumin (BSA), and then incubated with primary antibodies targeting Tspan9 $(1: 1,000$; Abcam, Cambridge, UK), ERK1/2 (1:1,000), p-ERK1/2 $(1: 2,000)$ (both from Cell Signaling Technology, Danvers, MA, USA) or tubulin (1:1,000; Beyotime, Jiangsu, China) in TBST containing $5 \%$ BSA overnight at $4^{\circ} \mathrm{C}$. Subsequently, incubation was conducted with the appropriate secondary antibodies for $1 \mathrm{~h}$ at room temperature. Reactive protein bands were visualized with BeyoECL Plus (Beyotime) and were detected using the Vilber enhanced chemiluminescence system (Vilber Lourmat, Marne la Vallée, France).

Cell viability assay. After trypsinization, the cells were seeded into 96 -well plates at a density of $0.2 \times 10^{4} /$ well for culture, and cell proliferation was measured by Cell Counting Kit- 8 (CCK-8; Dojindo, Tokyo, Japan) at 24, 48 and 72 h. Briefly, $10 \mu \mathrm{l}$ of CCK-8 solution was added to each well and incubation was performed for $2 \mathrm{~h}$ at $37^{\circ} \mathrm{C}$. Then the optical density (OD) was measured at $450 \mathrm{~nm}$ with a microplate spectrophotometer (BioTek Instruments, Winooski, VT, USA).

Cell cycle analysis. Cell cycle analysis was performed by a FACSCalibur cytometer (BD Biosciences, Franklin Lakes, NJ, USA) after staining the cells with propidium iodide (PI; Abcam). Cells were harvested by trypsinization, washed with phosphate-buffered saline (PBS), and fixed in $70 \%$ ethanol at $4^{\circ} \mathrm{C}$ overnight. On the following day, the fixed cells were washed with PBS, treated with RNase A $(50 \mu \mathrm{g} / \mathrm{ml})$ in PBS at $37^{\circ} \mathrm{C}$ for $20 \mathrm{~min}$ and then mixed with PI $(50 \mu \mathrm{g} / \mathrm{ml})$ for $30 \mathrm{~min}$ in the dark. After staining with PI had been completed, a minimum of 10,000 cells were counted for each sample by flow cytometry and the cell cycle profile was analyzed with FlowJo software (Tree Star, Inc., USA).

Wound-healing assay. Cells were seeded into 6-well plates at $90 \%$ confluency and incubated overnight for adherence. Then a wound was made along the center of each well by scratching the cell layer with the tip of a $200-\mu 1$ pipette (Axygen Scientific, Inc., Union City, CA, USA). Next, the wells were washed twice with PBS to remove loose cells and fresh medium was added. Images (x100 magnification) were captured at 0 and $24 \mathrm{~h}$ to assess cell migration into the wound. 
A

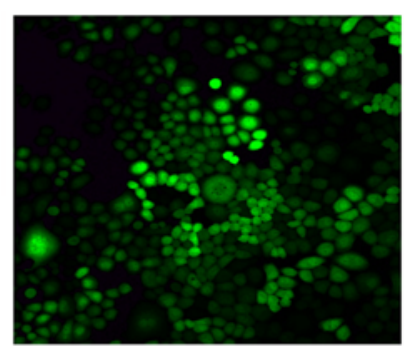

Tspan9

B

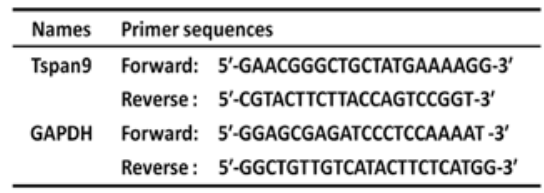

C

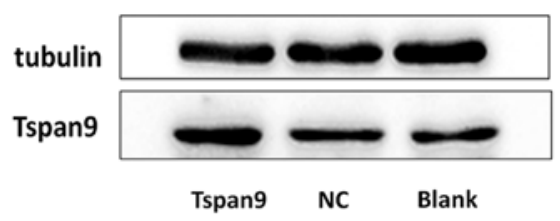

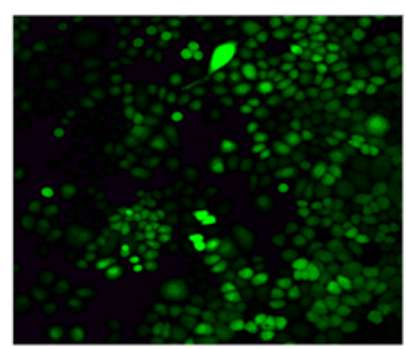

NC
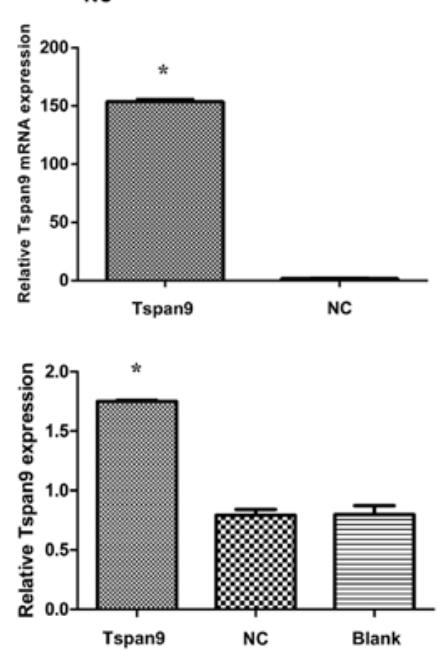

Figure 1. Construction of a stable cell line with overexpression of Tspan9. (A) Images show successfully infected SGC7901 cells with green fluorescent protein. Transduction efficiency of both groups was higher than $80 \%$. (B) Tspan9 mRNA expression level was significantly higher in the Tspan9 group (infected with LV-Tspan9) than that noted in the NC group (infected with LV-negative control) as detected by qRT-PCR. (C) Western blot analysis indicated that Tspan9 expression was markedly increased in the Tspan 9 group compared with that noted in the NC group, while there was no difference between the NC and blank group. Data represent mean $\pm \mathrm{SD}$. ${ }^{*} \mathrm{P}<0.05$.

Transwell assay for cell invasion and migration. Respectively, cell invasion and migration assays were conducted with or without Matrigel (Corning Inc., Corning, NY, USA). Cell invasion was measured using Matrigel-coated Transwell cell culture chambers ( $8-\mu \mathrm{m}$ pore size; Corning Inc.). After being maintained for $24 \mathrm{~h}$ in serum-free medium, the cells were trypsinized and resuspended in serum-free RPMI-1640 and placed in the upper chamber of the Transwell insert ( $5 \times 10^{4}$ cells/well), as RPMI-1640 containing 10\% FBS was added to the lower chamber. The plates were incubated in a humidified atmosphere with $95 \%$ air and $5 \% \mathrm{CO}_{2}$ at $37^{\circ} \mathrm{C}$ for 24 or $48 \mathrm{~h}$. Non-invasive cells in the upper chamber were removed by wiping with a cotton swab, and invasive cells were fixed with $4 \%$ formaldehyde in PBS and stained with $2 \%$ crystal violet in ethanol. Cells in the lower surface of the filter that penetrated through the Matrigel were counted under a light microscope at x200 magnification. Cell migration was determined as described for cell invasion assay except that the filter membrane was not coated with Matrigel. Cells located on the underside of the filter were counted under a light microscope at x200 magnification.

ELISA assay. The protein level of MMP-9 or UPA in the supernatant of the cells was assessed by MMP-9 or UPA ELISA kit (Neo Scientific, Cambridge, MA, USA). Cell supernatant was collected and centrifuged at $10,000 \mathrm{x}$ g for $10 \mathrm{~min}$ at $4^{\circ} \mathrm{C}$ to remove the cell debris. Then, $100 \mu \mathrm{l}$ of supernatant and standard were added to the wells in the supplied Neoplate while $100 \mu \mathrm{l}$ of PBS was added to the blank well, following the manufacturer's protocol. After adding $50 \mu \mathrm{l}$ of enzyme solution to each well (not blank well) and washed 5 times, substrate A $(50 \mu \mathrm{l})$ and substrate $\mathrm{B}(50 \mu \mathrm{l})$ were added to each well in order. Gentle mixing and incubation for $15 \mathrm{~min}$ at $37^{\circ} \mathrm{C}$ in dark was carried out and then $50 \mu \mathrm{l}$ of stop solution was added. Finally, optical density at $450 \mathrm{~nm}$ was read within $15 \mathrm{~min}$. The relative protein level of MMP-9 or uPA in each sample was determined by the standard curve.

Statistical analysis. SPSS 21 software (IBM, USA) was employed for statistical analysis. One-way ANOVA was conducted with Bonferroni's multiple comparison exact probability test, and the Student's t-test was used to compare continuous variables between two groups. Experiments with cell cultures were carried out at least in triplicate. Data are expressed as mean \pm standard deviation (SD). Statistical significance was accepted at $\mathrm{p}<0.05$.

\section{Results}

Tspan9 inhibits the proliferation of gastric cancer SGC7901 cells. We found that Tspan9 expression was lower in the SGC7901 cells (a poorly differentiated cell line) than that noted in the other cell lines. Accordingly, we chose this cell line for transfection with LV-Tspan9 (Fig. 1A) to better understand the role of Tspan9 in the development of GC. The effect of LV-Tspan9 was confirmed by real-time PCR (Fig. 1B) 
A

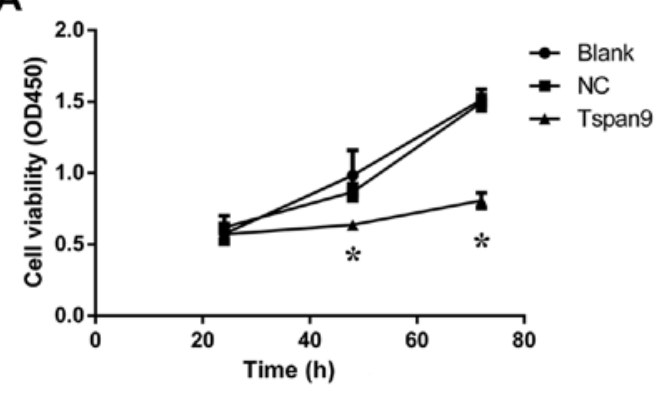

B

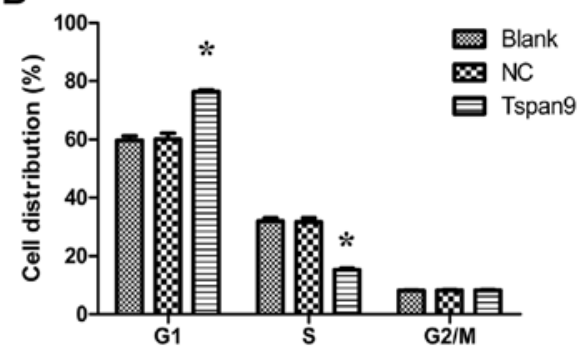

C

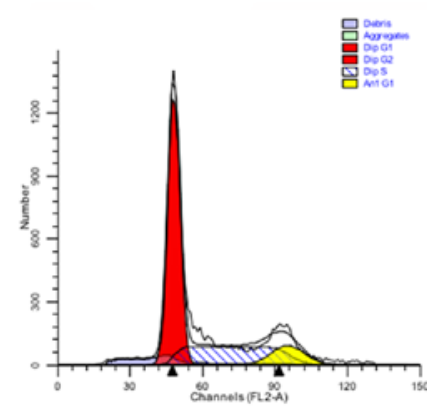

Blank

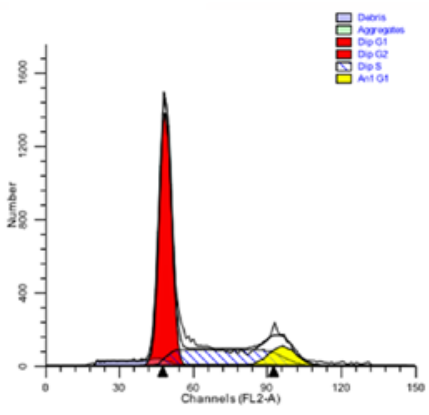

NC

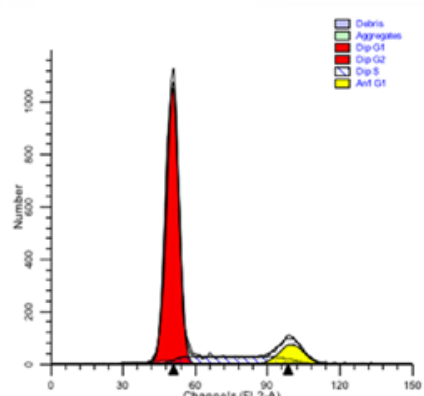

Tspan9

Figure 2. Tspan9 inhibits the proliferation of gastric cancer SGC7901 cells. (A) Tspan9 inhibits the cell viability of the SGC7901 cells via CCK-8 assays. (B and C) Cell cycle analysis revealed that Tspan9 arrested cell cycle progression by accumulation of cells in the G1 phase and reduced progression into the S phase. Data represent the mean \pm SD. ${ }^{*} \mathrm{P}<0.05$.
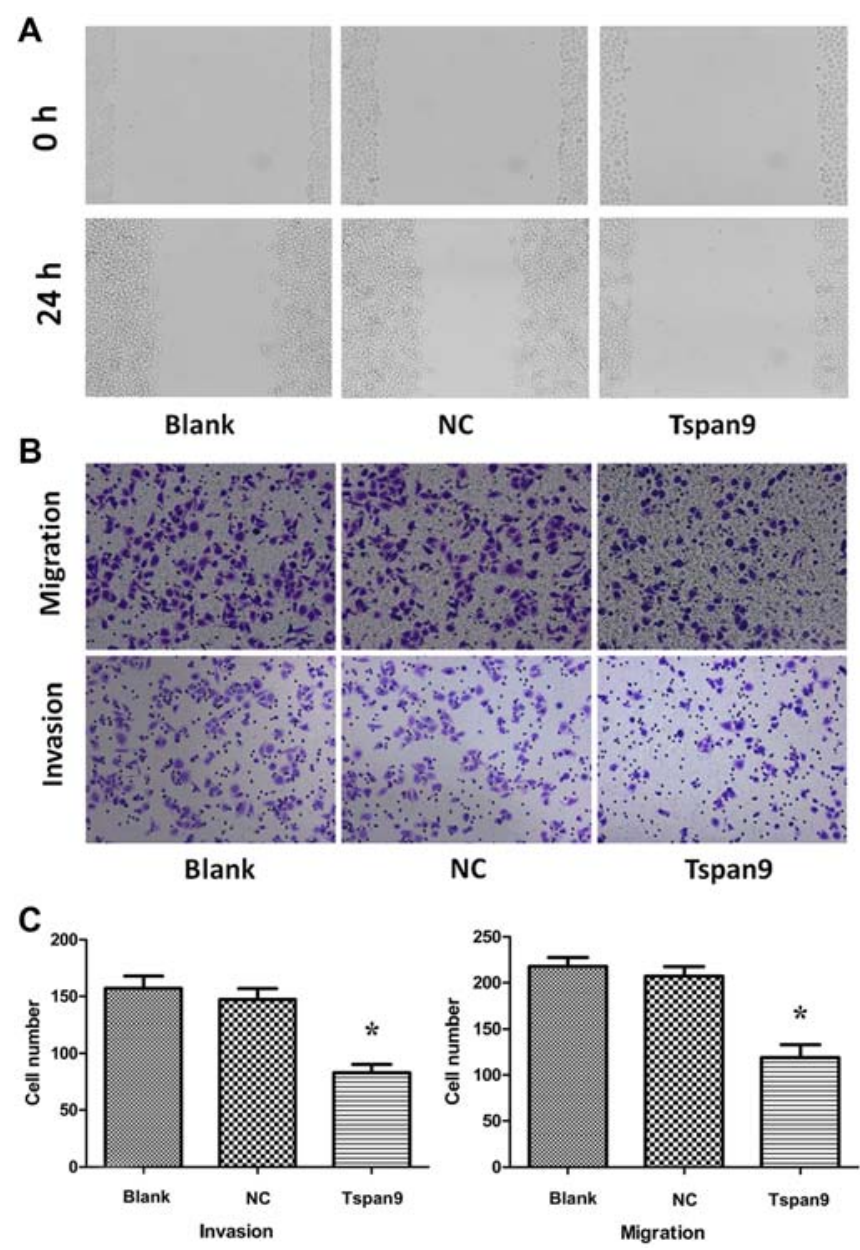

Figure 3. Tspan9 inhibits the migration and invasion of gastric cancer SGC7901 cells. (A) In the wound-healing assay, the residual wound was much wider in the Tspan9 group than that noted in the NC group at $24 \mathrm{~h}$. (B and C) Transwell assays revealed markedly decreased cell migration and invasion ability of the Tspan9 group compared with the NC group. Data represent mean \pm SD. ${ }^{*} \mathrm{P}<0.05$. 
A

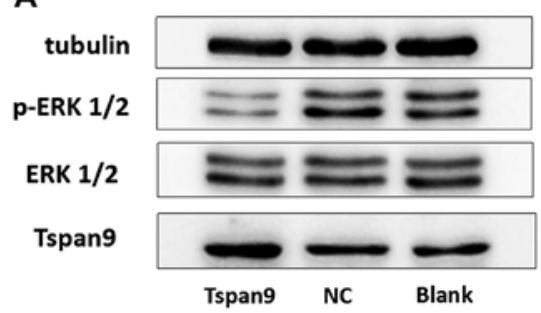

B

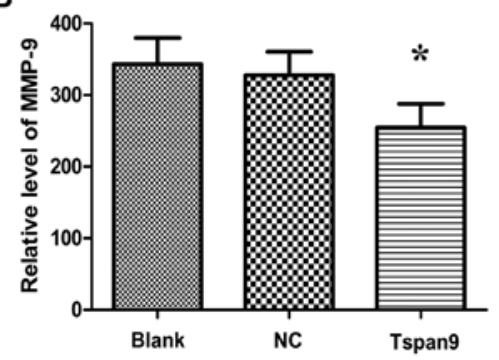

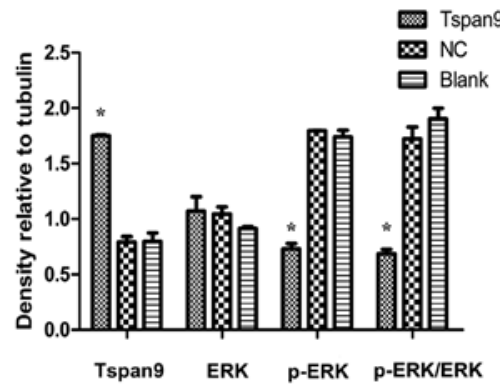

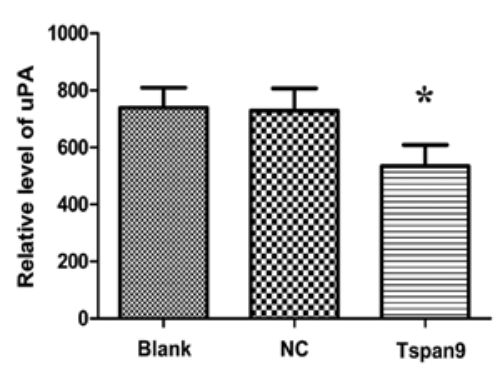

Figure 4. Tspan9 suppresses ERK1/2 activity and downregulates the secretion levels of MMP-9 and uPA. (A) Phosphorylation of ERK1/2 was significantly decreased in the Tspan 9 group based on western blotting. Tubulin was used as a loading control. (B) Secretion levels of MMP-9 and uPA were lower in the Tspan9 group than levels noted in the other groups. Data represent mean $\pm \mathrm{SD}$. ${ }^{*} \mathrm{P}<0.05$.

and western blot analysis (Fig. 1C). When the CCK-8 assay was used to assess cell viability over a period of 3 days, we found that cell viability was significantly lower in the Tspan9 group than that noted in the NC and blank groups, indicating that cell viability was suppressed by overexpression of Tspan9 (Fig. 2A). To analyze the mechanisms by which ectopic Tspan9 expression inhibited cell proliferation, flow cytometric analysis of the cell cycle was applied. As shown in Fig. 2B and C, the cells of the Tspan9 group were arrested in the G1 phase, accompanied by a significant reduction of cells in the $\mathrm{S}$ phase, compared with the other two groups. These results indicate that cell proliferation was markedly inhibited by high expression of Tspan9.

Tspan9 inhibits the migration and invasion of gastric cancer SGC7901 cells. To assess the effect of the upregulation of Tspan9 on cell motility, wound-healing assay and Transwell assays were performed. We found that fewer cells of the Tspan9 group migrated to the center of the wound in the woundhealing assay (Fig. 3A) compared to the NC and blank groups. In addition, overexpression of Tspan 9 obviously suppressed both the cell migration and invasion through Matrigel into the lower chamber in the Transwell assay (Fig. 3B and C). These findings support the notion that Tspan9 is involved in the modulation of the cell migration and invasion of GC cells.

Tspan9 suppresses ERK1/2 activity and downregulates the secretion levels of MMP-9 and $u P A$. The ERK1/2 pathways are known to play crucial roles in the promotion of cell survival. In order to elucidate whether the ERK1/2 pathway is involved in our experiments, we determined the levels of phosphorylated ERK1/2 (p-ERK1/2) and ERK1/2 by western blotting. Our results showed that overexpression of Tspan 9 inhibited p-ERK1/2 in the SGC7901 cells but had no influence on total ERK1/2 (Fig. 4A). As for metastasis-associated proteins, the secretion levels of MMP-9 and UPA were significantly lower in the Tspan9 group than levels in the other groups (Fig. 4B). These results indicate that Tspan 9 may inhibit the migration and invasion of gastric cancer SGC7901 cells by downregulating MMP-9 and uPA through the ERK1/2 pathway.

\section{Discussion}

The present study provides initial evidence concerning the role of Tspan9 in GC, showing that i) Tspan9 inhibits SGC7901 cell proliferation, migration and invasion, ii) Tspan9 is involved in the downregulation of the secretion of MMP-9 and uPA via the p-ERK1/2 pathway, and iii) Tspan9 is a potential tumor suppressor in patients with GC.

Tetraspanins are components of distinct multiprotein membrane microdomains where they interact with other tetraspanins and other membrane proteins such as integrins (particularly $\alpha 3 \beta 1, \alpha 4 \beta 1, \alpha 6 \beta 1$ and $\alpha 6 \beta 4$ isoforms), intracellular associated heterotrimeric $\mathrm{G}$ proteins, proteases, major histocompatibility complex (MHC) and immunoglobulin superfamily members depending on tetraspanin isoform, cellular context and other parameters $(8,20)$. And different tetraspanins interacting with various partners produce the variability and specificity among cell types (21). Thus, these tetraspanin-enriched microdomains (TEMs) contribute to the organization of cell surface proteins and can influence cell signal transduction $(22,23)$. It has been reported that Tspan 9 is relatively highly expressed in the megakaryocyte/platelet lineage and is a component of tetraspanin microdomains on the platelet surface. Tspan9 is markedly highly conserved during evolution, even relative to most other tetraspanins (11). As many tetraspanins (e.g., CD9, CD82, CD151 and tetraspanin 8) play crucial roles in carcinogenesis and cancer progression (9), we have reason to assume that Tspan9 may also participate in these processes.

In the present study, we firstly constructed a stable cell line overexpressing Tspan9 and then investigated the role of Tspan9 
in GC cells. Since tetraspanins have also been implicated in the control of cell proliferation directly or indirectly $(24,25)$, we wished to understand to what extent Tspan9 plays a role in cell proliferation of GC cells. The results presented in Fig. 2 indicate that Tspan9 is a potential driver of aberrant cell proliferation in GC. High expression of Tspan9 suppressed cell proliferation of SGC7901 cells compared with its controls. Furthermore, overexpression of Tspan 9 resulted in the arrest of the cell cycle progression as indicated by accumulation of cells in the G1 phase and reduced progression into the $\mathrm{S}$ phase compared with its negative control. Our data confirmed the regulatory function of Tspan9 in cancer development and progression. However, the downstream signaling pathways or specific factors which act on these biological functions of tumor cells, warrant further investigation. Identification and elucidation of the possible molecular mechanisms underlying them, also need further investigation.

Cancer cells can operate different migration programs under diverse environmental conditions (26). Cancer cell metastases, based on cancer cell migration and their invasion to surrounding tissues and vessels, require changes in locomotion-related genes and upstream signaling pathways $(27,28)$. In addition, different tetraspanin variants are involved in the regulation of cell motility and invasion. For instance, highly expressed CD151 was found to stimulate the migration of HeLa cells and promote migration and invasion in prostate cancer cell lines LNCaP and PC3 (12,29). In contrast, tetraspanin CD9 inhibited cell growth and motility of the human lung adenocarcinoma cell line MAC10 and the myeloma cell line ARH77 $(25,30)$. In our study, high expression of Tspan 9 depressed cell migration and invasion of the GC cell line SGC7901 by wound-healing and Transwell assays (Fig. 3). Thus, we speculated that distinct combinations of tetraspanins act in concert in distinct cell types to regulate and govern various aspects related to cancer cell motility, invasion and migration. Therefore, a comprehensive understanding of the effect of Tspan9 on cancer cell migration and invasion is needed.

In order to understand the mechanisms by which Tspan9 regulates the invasive properties of SGC7901 cells we focused on the expression of MMP-9 and UPA. On one hand, MMP-9 is a member of the matrix metalloproteinase family, which binds to zinc and acts on the extracellular matrix (ECM) to degrade type IV collagen in the basement membrane. Activation of MMPs has been detected in almost all types of human cancers, and are closely correlated to advanced tumor stage, increasing tumor invasion and metastasis. Metastasis occurs and the survival rate markedly decreases in GC patients as a result of loss of basement membrane integrity (31-34). Numerous studies indicate that inhibition of MMP expression or enzyme activity can be used as early targets for preventing cancer metastasis $(19,35,36)$. As a member of the serine protease family, uPA plays a crucial role in the decomposition of basement membranes, and the activation of the uPA/ uPAR/plasmin proteolytic network plays a key role in tumor invasion and dissemination of various malignancies (37-39). The levels of UPA and UPAR expression serve as prognostic markers in various malignancies, and high levels of expression are often associated with a poor prognosis (40). Furthermore, many tetraspanins participate in the regulation of MMPs and UPA by recruitment of specific signals and by activating or inhibiting diverse signaling pathways (e.g., PI3K and Ras-Raf-MAPK pathway) (9). Based on the above investigations, MMP-9 and uPA, two metastasis-associated proteins, were selected in our study. Our result showed that Tspan9 downregulated the secretion levels of MMP-9 and uPA in the SGC7901 cells significantly, confirming that Tspan9 may be a tumor suppressor reducing the motility and invasiveness of GC cells.

Numerous studies have shown that MAPKs (JNK1/2, ERK1/2, and p38) are involved in cancer cell proliferation, migration, invasion, and changes in MMP or uPA activity (19,41-43). The ERK1/2 pathway plays an important role in the invasion or metastasis of a number of tumors, such as oral cancer, hepatocellular carcinoma, prostate cancer and lung cancer $(28,44,45)$. In the present study, we observed that the ERK1/2 signaling pathway mediated expression of MMP-9 and UPA in response to Tspan9, which further contributed to cellular motility of the human GC SGC7901 cells. In conclusion, the anti-proliferation, anti-migration, and anti-invasion effects of Tspan9 are likely based on its inactivation of the ERK1/2 pathway in SGC7901 cells, as shown in our study. Our evidence suggests that downregulation of ERK1/2 signaling pathway by Tspan9 is an important mechanism underlying cancer progression, and may serve as a potential treatment for modulating this pathway in cancers. In addition, the specific molecular mechanisms of Tspan9 and corresponding TEMs require elucidation. Much research is needed before Tspan9 can be used as a molecular-target in the therapy for GC.

In summary, Tspan9 may be an important tumor suppressor of GC. As shown in our research, high expression of Tspan9 significantly inhibited the proliferation, migration and invasion of human gastric cancer SGC7901 cells via inactivation of the ERK1/2 pathway. Better understanding of the precise molecular mechanism in the Tspan9-mediated signaling pathway may help design an effective therapeutic modality to control GC.

\section{Acknowledgements}

This study was supported by the National Natural Science Foundation of China (81472338) and A Project of Shandong Province Higher Educational Science and Technology Program (J15LL58).

\section{References}

1. Carcas LP: Gastric cancer review. J Carcinog 13: 14, 2014.

2. Parkin DM, Ferlay J, Curado MP, Bray F, Edwards B, Shin HR and Forman D: Fifty years of cancer incidence: CI5 I-IX. Int J Cancer 127: 2918-2927, 2010.

3. Lee AJ, Endesfelder D, Rowan AJ, Walther A, Birkbak NJ, Futreal PA, Downward J, Szallasi Z, Tomlinson IP, Howell M, et al: Chromosomal instability confers intrinsic multidrug resistance. Cancer Res 71: 1858-1870, 2011.

4. Simons K and Toomre D: Lipid rafts and signal transduction. Nat Rev Mol Cell Biol 1: 31-39, 2000.

5. Hemler ME: Targeting of tetraspanin proteins - potential benefits and strategies. Nat Rev Drug Discov 7: 747-758, 2008.

6. Claas C, Stipp CS and Hemler ME: Evaluation of prototype transmembrane 4 superfamily protein complexes and their relation to lipid rafts. J Biol Chem 276: 7974-7984, 2001.

7. Yáñez-Mó M, Barreiro O, Gordon-Alonso M, Sala-Valdés M and Sánchez-Madrid F: Tetraspanin-enriched microdomains: A functional unit in cell plasma membranes. Trends Cell Biol 19: 434-446, 2009. 
8. Mazzocca A, Birgani MT, Sabbà C and Carloni V: Tetraspanin-enriched microdomains and hepatocellular carcinoma progression. Cancer Lett 351: 23-29, 2014.

9. Zöller M: Tetraspanins: Push and pull in suppressing and promoting metastasis. Nat Rev Cancer 9: 40-55, 2009.

10. Kovalenko OV, Metcalf DG, DeGrado WF and Hemler ME: Structural organization and interactions of transmembrane domains in tetraspanin proteins. BMC Struct Biol 5: 11, 2005.

11. Protty MB, Watkins NA, Colombo D, Thomas SG, Heath VL, Herbert JM, Bicknell R, Senis YA, Ashman LK, Berditchevski F, et al: Identification of Tspan9 as a novel platelet tetraspanin and the collagen receptor GPVI as a component of tetraspanin microdomains. Biochem J 417: 391-400, 2009.

12. Sadej R, Grudowska A, Turczyk L, Kordek R and Romanska HM CD151 in cancer progression and metastasis: A complex scenario. Lab Invest 94: 41-51, 2014.

13. Murayama Y, Oritani K and Tsutsui S: Novel CD9-targeted therapies in gastric cancer. World J Gastroenterol 21: 3206-3213, 2015.

14. Cakarovski K, Leung JY, Restall C, Carin-Carlson A, Yang E, Perlmutter P, Anderson R, Medcalf R and Dear AE: Novel inhibitors of urokinase-type plasminogen activator and matrix metalloproteinase expression in metastatic cancer cell lines. Int J Cancer 110: 610-616, 2004.

15. Toda D, Ota $T$, Tsukuda $K$, Watanabe $K$, Fujiyama $T$, Murakami M, Naito M and Shimizu N: Gefitinib decreases the synthesis of matrix metalloproteinase and the adhesion to extracellular matrix proteins of colon cancer cells. Anticancer Res 26: 129-134, 2006

16. Yang SF, Hsieh YS, Lin CL, Hsu NY, Chiou HL, Chou FP and Chu SC: Increased plasma levels of urokinase plasminogen activator and matrix metalloproteinase-9 in nonsmall cell lung cancer patients. Clin Chim Acta 354: 91-99, 2005.

17. Mook OR, Frederiks WM and Van Noorden CJ: The role of gelatinases in colorectal cancer progression and metastasis. Biochim Biophys Acta 1705: 69-89, 2004.

18. Hwang ES and Lee HJ: Benzyl isothiocyanate inhibits metalloproteinase-2/-9 expression by suppressing the mitogen-activated protein kinase in SK-Hep1 human hepatoma cells. Food Chem Toxicol 46: 2358-2364, 2008

19. Lai KC, Huang AC, Hsu SC, Kuo CL, Yang JS, Wu SH and Chung JG: Benzyl isothiocyanate (BITC) inhibits migration and invasion of human colon cancer HT29 cells by inhibiting matrix metalloproteinase-2/-9 and urokinase plasminogen (uPA) through PKC and MAPK signaling pathway. J Agric Food Chem 58: 2935-2942, 2010.

20. Hölters S, Anacker J, Jansen L, Beer-Grondke K, Dürst M and Rubio I: Tetraspanin 1 promotes invasiveness of cervical cancer cells. Int J Oncol 43: 503-512, 2013.

21. Carloni V, Mazzocca A, Mello T, Galli A and Capaccioli S: Cell fusion promotes chemoresistance in metastatic colon carcinoma. Oncogene 32: 2649-2660, 2013.

22. Rubinstein E, Le Naour F, Lagaudrière-Gesbert C, Billard M, Conjeaud $\mathrm{H}$ and Boucheix C: CD9, CD63, CD81, and CD82 are components of a surface tetraspan network connected to HLA-DR and VLA integrins. Eur J Immunol 26: 2657-2665, 1996.

23. Berditchevski F, Odintsova E, Sawada S and Gilbert E: Expression of the palmitoylation-deficient CD151 weakens the association of alpha 3 beta 1 integrin with the tetraspanin-enriched microdomains and affects integrin-dependent signaling. J Biol Chem 277: 36991-37000, 2002

24. Chen L, Yuan D, Zhao R, Li H and Zhu J: Suppression of TSPAN1 by RNA interference inhibits proliferation and invasion of colon cancer cells in vitro. Tumori 96: 744-750, 2010.

25. Ovalle S, Gutiérrez-López MD, Olmo N, Turnay J, Lizarbe MA, Majano P, Molina-Jiménez F, López-Cabrera M, Yáñez-Mó M, Sánchez-Madrid F, et al: The tetraspanin CD9 inhibits the proliferation and tumorigenicity of human colon carcinoma cells. Int J Cancer 121: 2140-2152, 2007

26. Friedl $P$ and Alexander S: Cancer invasion and the microenvironment: Plasticity and reciprocity. Cell 147: 992-1009, 2011.

27. Sahai E: Mechanisms of cancer cell invasion. Curr Opin Genet Dev 15: 87-96, 2005.

28. Yang J, Kuang XR, Lv PT and Yan XX: Thymoquinone inhibits proliferation and invasion of human nonsmall-cell lung cancer cells via ERK pathway. Tumour Biol 36: 259-269, 2015.
29. Ang J, Fang BL, Ashman LK and Frauman AG: The migration and invasion of human prostate cancer cell lines involves CD151 expression. Oncol Rep 24: 1593-1597, 2010.

30. Longo N, Yáñez-Mó M, Mittelbrunn M, de la Rosa G, Muñoz ML, Sánchez-Madrid F and Sánchez-Mateos P: Regulatory role of tetraspanin CD9 in tumor-endothelial cell interaction during transendothelial invasion of melanoma cells. Blood 98: 3717-3726, 2001

31. Kubben FJ, Sier CF, van Duijn W, Griffioen G, Hanemaaijer R, van de Velde CJ, van Krieken JH, Lamers CB and Verspaget HW: Matrix metalloproteinase-2 is a consistent prognostic factor in gastric cancer. Br J Cancer 94: 1035-1040, 2006.

32. Chu D, Zhang Z, Li Y, Zheng J, Dong G, Wang W and Ji G: Matrix metalloproteinase-9 is associated with disease-free survival and overall survival in patients with gastric cancer. Int J Cancer 129: 887-895, 2011.

33. Tang Y, Zhu J, Chen L, Chen L, Zhang S and Lin J: Associations of matrix metalloproteinase-9 protein polymorphisms with lymph node metastasis but not invasion of gastric cancer. Clin Cancer Res 14: 2870-2877, 2008

34. Verma S, Kesh K, Gupta A and Swarnakar S: An overview of matrix metalloproteinase 9 polymorphism and gastric cancer risk. Asian Pac J Cancer Prev 16: 7393-7400, 2015.

35. Akter H, Park M, Kwon OS, Song EJ, Park WS and Kang MJ: Activation of matrix metalloproteinase-9 (MMP-9) by neurotensin promotes cell invasion and migration through ERK pathway in gastric cancer. Tumour Biol 36: 6053-6062, 2015.

36. Guruvayoorappan C and Kuttan G: Amentoflavone inhibits experimental tumor metastasis through a regulatory mechanism involving MMP-2, MMP-9, prolyl hydroxylase, lysyl oxidase, VEGF, ERK-1, ERK-2, STAT-1, NM23 and cytokines in lung tissues of C57BL/6 mice. Immunopharmacol Immunotoxicol 30: 711-727, 2008.

37. Alfano D, Votta G, Schulze A, Downward J, Caputi M, Stoppelli MP and Iaccarino I: Modulation of cellular migration and survival by c-Myc through the downregulation of urokinase (uPA) and uPA receptor. Mol Cell Biol 30: 1838-1851, 2010.

38. Ahmad A, Kong D, Wang Z, Sarkar SH, Banerjee S and Sarkar FH: Down-regulation of uPA and UPAR by 3,3'-diindolylmethane contributes to the inhibition of cell growth and migration of breast cancer cells. J Cell Biochem 108: 916-925, 2009.

39. Ahmad A, Kong D, Sarkar SH, Wang Z, Banerjee S and Sarkar FH: Inactivation of uPA and its receptor uPAR by 3,3'-diindolylmethane (DIM) leads to the inhibition of prostate cancer cell growth and migration. J Cell Biochem 107: 516-527, 2009.

40. Duffy MJ, Maguire TM, McDermott EW and O'Higgins N: Urokinase plasminogen activator: A prognostic marker in multiple types of cancer. J Surg Oncol 71: 130-135, 1999.

41. Chou YC, Chang MY, Wang MJ, Yu FS, Liu HC, Harnod T, Hung $\mathrm{CH}$, Lee HT and Chung JG: PEITC inhibits human brain glioblastoma GBM 8401 cell migration and invasion through the inhibition of uPA, Rho A, and Ras with inhibition of MMP-2, -7 and -9 gene expression. Oncol Rep 34: 2489-2496, 2015.

42. Ma CY,Ji WT, Chueh FS, Yang JS, Chen PY, Yu CC and Chung JG: Butein inhibits the migration and invasion of SK-HEP-1 human hepatocarcinoma cells through suppressing the ERK, JNK, p38, and uPA signaling multiple pathways. J Agric Food Chem 59: 9032-9038, 2011

43. Hsu HH, Hu WS, Lin YM, Kuo WW, Chen LM, Chen WK, Hwang JM, Tsai FJ, Liu CJ and Huang CY: JNK suppression is essential for 17 $\beta$-estradiol inhibits prostaglandin E2-Induced uPA and MMP-9 expressions and cell migration in human LoVo colon cancer cells. J Biomed Sci 18: 61, 2011.

44. Lin CW, Chen PN, Chen MK, Yang WE, Tang CH, Yang SF and Hsieh YS: Kaempferol reduces matrix metalloproteinase-2 expression by down-regulating ERK1/2 and the activator protein-1 signaling pathways in oral cancer cells. PLoS One 8: $\mathrm{e} 80883,2013$

45. Lee SH, Jaganath IB, Manikam R and Sekaran SD: Inhibition of Raf-MEK-ERK and hypoxia pathways by Phyllanthus prevents metastasis in human lung (A549) cancer cell line. BMC Complement Altern Med 13: 271, 2013. 